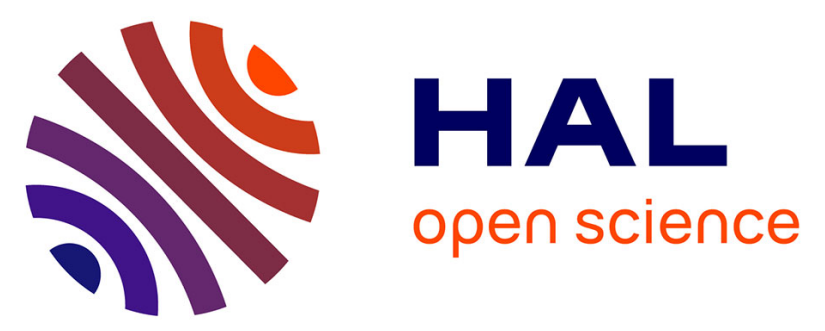

\title{
IMC, tour de taille et transplantation rénale : contraintes, réalités et enjeux pour un centre spécialisé de l'obésité. Revue de la littérature
}

Jean-Baptiste Bonnet, Dan Grey, Ashley Agbanglanon, Laetitia Galibert, Danielson Rakotoarivony, Marianne Vidal, Christophe Gourc, Vincent Attalin, Antoine Avignon

\section{To cite this version:}

Jean-Baptiste Bonnet, Dan Grey, Ashley Agbanglanon, Laetitia Galibert, Danielson Rakotoarivony, et al.. IMC, tour de taille et transplantation rénale : contraintes, réalités et enjeux pour un centre spécialisé de l'obésité. Revue de la littérature. Nutrition Clinique et Métabolisme, 2020, 10.1016/j.nupar.2020.10.003 . hal-03009377

\section{HAL Id: hal-03009377 https://hal.science/hal-03009377}

Submitted on 18 Nov 2020

HAL is a multi-disciplinary open access archive for the deposit and dissemination of scientific research documents, whether they are published or not. The documents may come from teaching and research institutions in France or abroad, or from public or private research centers.
L'archive ouverte pluridisciplinaire HAL, est destinée au dépôt et à la diffusion de documents scientifiques de niveau recherche, publiés ou non, émanant des établissements d'enseignement et de recherche français ou étrangers, des laboratoires publics ou privés. 


\title{
IMC, tour de taille et transplantation rénale : contraintes, réalités et enjeux pour un centre spécialisé de l'obésité. Revue de la littérature
}

\author{
BMI, waist circumference and kidney transplantation: Constraints, realities and \\ issues for a specialized obesity center
}

\author{
Jean-Baptiste Bonnet ${ }^{\mathrm{a}, *, \mathrm{~b}}$, Dan Grey ${ }^{\mathrm{a}}$, Ashley Agbanglanon ${ }^{\mathrm{a}}$, Laetitia Galibert ${ }^{\mathrm{a}}$, \\ Danielson Rakotoarivony ${ }^{\mathrm{a}}$, Marianne Vidal ${ }^{\mathrm{a}}$, Christophe Gourc ${ }^{\mathrm{a}}$, Vincent Attalin ${ }^{\mathrm{a}}$, \\ Antoine Avignon ${ }^{\mathrm{a}, \mathrm{b}}$ \\ a Unité Transversale de Nutrition (UTN), CHU de Montpellier, 371, avenue du Doyen-Gaston-Giraud, 34295 Montpellier cedex 5, France \\ ${ }^{\mathrm{b}}$ Université de Montpellier, Montpellier, France
}

\author{
Mots clés : \\ Transplantation rénale \\ Obésité \\ Tour de taille \\ Nutrition \\ Chirurgie bariatrique
}

\section{R É S U M É}

Si les personnes avec une insuffisance rénale chronique terminale et vivant en situation d'obésité ont un pronostic considérablement amélioré par la transplantation rénale, elles sont encore confrontées à de nombreux freins à l'accès à la greffe. L'effet de l'obésité sur la survie du greffon et la survie toutes causes est de moins en moins démontré. Les questions sur la gestion des immunosuppresseurs, des diabètes post transplantation et des syndromes d'hyperfiltration glomérulaire restent, cependant, toujours un enjeu. Néanmoins, la barrière principale est celle du geste chirurgical. Les complications opératoires restent un frein à l'inscription sur liste. Parallèlement, les programmes d'amaigrissement en dialyse avant inscription se confrontent à la surmortalité des cohortes. La question de la chirurgie bariatrique pour les personnes avec les IMC les plus importants peut être une solution qui doit encore être consolidée. Les programmes de réentraînement physique en dialyse sont aussi des pistes de recherche clinique dont l'exploration doit se poursuivre. Enfin, L'arrivée du robot pour la chirurgie pourrait permettre de lever certaines barrières. Ces notions chirurgicales nous amènent, par ailleurs, à nous demander si l'IMC est le bon indicateur. Le tour de taille, reflet du tablier abdominal, n'est-il finalement pas l'élément bloquant du chirurgien ? Quoi qu'il en soit, la Haute Autorité de Santé ne fixe, aujourd'hui, une limite à la transplantation rénale que pour des IMC au-dessus de $50 \mathrm{~kg} / \mathrm{m}^{2}$. Cette règle est encore loin d'être une réalité pour les personnes vivant en situation d'obésité en France et dans le monde.

\section{Keywords:}

Kidney transplantation

Obesity

Nutrition

Waist size

Bariatric surgery

\section{A B S T R A C T}

While patients with an end-stage renal disease and living with obesity have a significantly improved prognosis with kidney transplantation, they still face many barriers to access to it. Obesity effect on graft survival and all-cause survival is less and less established. However, questions about immunosuppressive drugs management, post-transplant diabetes and glomerular hyperfiltration syndromes remain an issue. Nevertheless, surgical gesture remains the main barrier. Complications from surgery still restrain registration. At the same time, pre-registration weight-loss programs under dialysis face an excess of mortality. Bariatric surgery issue for people with the highest BMIs might be a solution that still needs more research. Exercise rehabilitation during dialysis programs are also major avenues that need

\footnotetext{
* Auteur correspondant.

Adresse e-mail : jean-baptiste-bonnet@chu-montpellier.fr (J.-B. Bonnet).
} 
further explorations. Finally, robot-assisted surgery arrival could remove many restrictions. These surgical notions also lead us to wonder whether BMI is the right indicator. Isn't the waist circumference, a direct reflection of the abdominal apron, the blocking element for surgery? In any case, the French National Authority for Health currently only sets a limitation on kidney transplantation for BMIs above $50 \mathrm{~kg} / \mathrm{m}^{2}$. This rule is still far from being a reality for people living with obesity in France and around the world.

\section{Introduction}

La transplantation rénale est le traitement de référence de l'insuffisance rénale chronique terminale (IRCT). Elle permet de s'affranchir de la dialyse et d'améliorer significativement la qualité et l'espérance de vie. Cependant, la transplantation rénale est limitée, faute de disponibilité des greffons. Limite que ni les nouvelles procédures Maastricht [1], ni les programmes de donneur vivant n'ont pour l'instant pu lever. Les personnes en situation d'obésité représentent aujourd'hui une part substantielle de la population éligible à la transplantation rénale. Elles font cependant fréquemment les frais de la pénurie d'organes en étant récusées pour la transplantation du seul fait de leur index de masse corporelle (IMC). Les derniers critères érigés par la Haute Autorité de santé (HAS) datant de 2015 considèrent pourtant que la contre-indication à la transplantation n'est valable que pour les obésités les plus sévères avec un IMC au-delà de $50 \mathrm{~kg} / \mathrm{m}^{2}$, ce qui ne représente qu'une minorité des personnes vivant avec une obésité. Nous faisons ici le point sur la situation épidémiologique, les problématiques médicales et les perspectives qui s'offrent aux personnes vivant avec une obésité.

\section{La place des personnes en situation d'obésité dans la transplantation rénale}

La transplantation rénale améliore drastiquement le pronostic de survie des patients en dialyse tout en réduisant considérablement les coûts dès la deuxième année de prise en charge [2]. Le suivi des populations greffées ou en attente de transplantation présente l'avantage d'être structuré et surveillé par des cohortes nationales ou internationales permettant d'évaluer le pronostic à long terme. Il faut tout de même prendre en compte le biais de sélection d'une situation de pénurie.

Toute personne à risque, donneuse ou receveuse, se verra presque systématiquement sous représentée dans les listes de transplantation et, par conséquent, dans ces cohortes qui guident nos décisions. En effet, le médecin transplanteur cherche à donner le bon greffon à la bonne personne en valorisant au maximum une ressource rare.

Tout d'abord, il est constaté que quel que soit l'IMC de la personne bénéficiant d'une dialyse, la transplantation améliore son pronostic vital [3]. Pour celles dont l'IMC est supérieur à $40 \mathrm{~kg} / \mathrm{m}^{2}$, des cohortes américaines ont révélé une amélioration en termes de survie de 48 \% [4]. De ce fait, selon l'épidémiologie occidentale, et plus particulièrement américaine, la proportion des personnes inscrites sur liste avec un IMC supérieur à $35 \mathrm{~kg} / \mathrm{m}^{2}$ s'accroît chaque année depuis 40 ans. Aux États-Unis, elle est d'ailleurs passée de $1 \%$ à plus de $7 \%$ depuis le début des années 2000 . Le nombre de personnes avec un IMC supérieur à $40 \mathrm{~kg} / \mathrm{m}^{2}$ reste plus stable [5] ce qui témoigne d'un défaut d'inscription sur liste au regard de l'épidémiologie du pays. Toujours aux Etats-Unis, les personnes avec un IMC supérieur à $25 \mathrm{~kg} / \mathrm{m}^{2}$ représentaient déjà plus de $60 \%$ des personnes sur liste d'attente de transplantation au tournant du siècle [6]. En France, le registre REIN 2013 rapporte une préva- lence de l'obésité à 23,9\% à l'initiation de la dialyse (versus $15 \%$ en 2009 en France en population générale selon OBEPI) [7].

Néanmoins, une fois sur liste, la probabilité d'être greffé est inversement proportionnelle à l'IMC avec des effets seuils importants au-dessus de $35 \mathrm{~kg} / \mathrm{m}^{2}$ puis $40 \mathrm{~kg} / \mathrm{m}^{2}$ [5]. Ainsi, l'IMC est inversement corrélé à la probabilité d'être appelé une fois sur la liste. Cette probabilité est divisée par deux pour ceux dont l'IMC se trouve au-dessus de $40 \mathrm{~kg} / \mathrm{m}^{2}$. Si le patient est contacté, il existe à nouveau un lien positif entre l'IMC et la probabilité d'être finalement refusé par le centre transplanteur. En 2018, dans une étude espagnole au sein des centres de dialyse, Toapanta-Gaibor et al. montraient que l'IMC était la première cause de refus d'inscription sur liste (30\% de la population) [8]. En France, la HAS estime que l'IMC n'est une contre-indication pour la transplantation qu'à partir d'un IMC de $50 \mathrm{~kg} / \mathrm{m}^{2}$ [9]. Enfin, aux États-Unis Suresh et al. rapportaient en 2020 que la première cause de demande de perte de poids exprimée par les patients en dialyse vers les équipes de diététique était la volonté d'être inscrit sur liste [10].

Pour relativiser ces notions, il est intéressant de noter qu'un IMC élevé reste un facteur protecteur en dialyse. La Dialysis Outcomes and Practice Patterns Study (DOPPS) l'a montré, même sur des cohortes anciennes, aux Etats-Unis, mais aussi en Europe [11]. D'un autre côté, les données sur la survie, une fois greffé, ne sont pas consensuelles. Le registre national hollandais décrit par Aalten et al. sur la période 1996-2006 montre des courbes de survie (mortalité toutes causes) et de survie du greffon (rejet aigu inclus) en " $U$ " avec des augmentations de mortalité et de perte de greffon chez les IMC bas et hauts [12]. Le registre australo-néo-zélandais sur une période similaire (1991-2004) ne montrait, lui, aucun effet de l'IMC sur ces mêmes critères qu'étaient le décès et la perte de greffon [13]. Ceci peut également être constaté sur une série de registres nationaux de qualité qui montrent alternativement un effet statistique de l'obésité et une absence de lien quel que soit l'excès pondéral. Seules les cohortes antérieures à 2003 semblent montrer une augmentation de la mortalité liée à un IMC élevé.

Les équipes de transplantation rénale sollicitent régulièrement les centres spécialisés de l'obésité (CSO) dans le cadre de l'inscription sur liste de transplantation des personnes en situation d'obésité avec une demande de perte de poids en vue de l'intervention. L'objectif de cette revue est de dresser un état des lieux des arguments en faveur d'une perte de poids avant transplantation rénale pour les personnes en situation d'obésité.

\section{Impact de l'obésité sur la chirurgie}

La transplantation rénale se pratiquant au niveau des vaisseaux iliaques, le site opératoire, profond, est impacté par l'épaisseur du tablier abdominal, ce qui en fait le premier frein pour le chirurgien. L'IMC est ainsi corrélé au temps opératoire [14], au risque de hernie et au risque d'infection du site opératoire [15]. Une solution de plus en plus proposée actuellement est la chirurgie assistée par robot. Celle-ci présente l'avantage, via la longueur de ses tiges, de pouvoir intervenir profondément dans les tissus des patients sans pour autant élargir l'incision de laparotomie et donc de limiter les 
risques d'infection et de hernie. Cette solution apporte un certain consensus dans les publications récentes en termes de réduction des complications chirurgicales sans altérer la survie et la fonctionnalité du greffon dans le suivi post-opératoire [16,17]. Bien évidemment ce type de technique est conditionné à un investissement financier non négligeable du centre transplanteur et à la présence d'un chirurgien formé et entraîné à l'outil.

\section{Impact de l'obésité sur le greffon rénal}

Au-delà de la question chirurgicale, l'obésité pose un certain nombre de questions sur la survie du greffon. Il y a tout d'abord les complications rénales classiques de l'obésité. Notons l'hyperfiltration glomérulaire avec la hyalinose segmentaire et focale [18] ainsi que le lien entre IMC, diabète et néphropathie diabétique. Un IMC au-dessus de $30 \mathrm{~kg} / \mathrm{m}^{2}$ serait lié à doublement de la prévalence de la protéinurie, témoin de l'atteinte glomérulaire dans ce contexte [19]. Ni l'obésité ni le diabète ne disparaissent avec la transplantation. Le risque de récidive de la néphropathie initiale est donc important et les réflexions pour la transplantation rénale sont les mêmes que celles qui ont lieu pour le risque de récidive posttransplantation hépatique de la stéatose hépatique non alcoolique (Non-Alcoholic SteatoHepatitis [NASH]). Il est à noter que, contrairement à la transplantation hépatique, la transplantation rénale ne bénéficie pas de l'immunotolérance relative hépatique et est donc tributaire d'une immunosuppression plus appuyée, notamment par la corticothérapie au long cours. Cette corticothérapie s'ajoute à la fin du stress organique qu'est la dialyse et conduit à une prise de poids quasi constante au décours de la transplantation rénale [20]. L'augmentation de l'IMC chez le patient déjà obèse ne fait que renforcer les craintes citées ci-dessus sur l'hyperfiltration glomérulaire et la néphropathie diabétique.

À ces complications rénales connues de l'obésité viennent s'ajouter celles spécifiques à la transplantation telles que la reprise de fonction du greffon observée plus tardivement [21], la survenue de diabètes post transplantation (New Onset Diabetes after transplantation : NODAT) ou la décompensation d'un diabète de type 2 préexistant [22].

\section{Les difficultés d'équilibre des immunosuppresseurs}

Flabouris et al. ont présenté un travail reprenant les problématiques de l'immunosuppression après transplantation rénale chez la personne vivant avec une obésité [23]. Dans cette analyse du registre australo-néo-zélandais, les immunosuppresseurs sont en moyenne sous dosés chez les personnes avec un IMC au-dessus de $25 \mathrm{~kg} / \mathrm{m}^{2}$. Ce constat vaut pour le tacrolimus, la cyclosporine ou le mycophénolate mofétil. Parallèlement, après ajustement sur les comorbidités, les personnes avec un IMC au-dessus de $25 \mathrm{~kg} / \mathrm{m}^{2}$ apparaissent comme faisant plus de rejets aigus à 6 mois. L'ajustement sur les immunosuppresseurs annule ce sur-risque, confortant la suspicion d'implication du sous-dosage en immunosuppresseurs. Ceci est confirmé par un taux d'infection dans la moyenne sans ajustement, mais augmenté avec ajustement sur les immunosuppresseurs, confirmant l'hypothèse d'une immunité globalement préservée chez cette population. Les résultats sur les cancers, de la peau notamment, n'étaient pas concordants mais nous sommes peut-être face à un biais de diagnostic des cancers chez la personne vivant avec une obésité.

Quoi qu'il en soit, que ce soit avec ou sans ajustement sur les comorbidités et les immunosuppresseurs, les auteurs ne retrouvaient pas de sur-risque de perte de greffon ou de mortalité en fonction de l'IMC. Il est vrai que les progrès sur les immunosuppresseurs au cours de ces 30 dernières années concernent surtout le rejet aigu et beaucoup moins le rejet chronique pour lequel les chiffres plafonnent depuis plusieurs décennies.

\section{Perdre du poids avant une transplantation rénale}

Nous avons donc constaté que la transplantation améliorerait le pronostic des personnes en situation d'obésité en IRCT mais que leur IMC était, en lui-même, un frein à l'inscription sur liste et à la transplantation rénale. Il parait maintenant intuitif de poser la question de la perte de poids avant le geste opératoire. Toujours sur le registre australien et néo-zélandais, Brilleman et al. ont étudié le pronostic des personnes perdant du poids sur la liste d'attente de transplantation (et donc en dialyse) et l'effet sur l'accessibilité à la transplantation pour ces personnes. Ils retrouvent la gradation de l'accessibilité à la transplantation en fonction de l'IMC. Ils constatent aussi qu'un profil de personnes passant d'un IMC de $37 \mathrm{~kg} / \mathrm{m}^{2}$ à un IMC de $18 \mathrm{~kg} / \mathrm{m}^{2}$ améliore fondamentalement la probabilité d'avoir une transplantation à 5 ans. Cependant, parallèlement à cela, le taux de mortalité en dialyse grimpe pour atteindre 60 \% à 5 ans sur liste d'attente pour cette même population voyant son IMC s'effondrer en dialyse. Sur une perte de poids un peu moins significative avec un IMC passant de 32 à $25 \mathrm{~kg} / \mathrm{m}^{2}$, la probabilité d'être transplanté n'est pas forcément augmentée. En revanche, l'augmentation des taux de mortalité chez ces patients sur liste est toujours largement significative, atteignant $40 \%$ à 5 ans (pour un taux moyen de $10 \%$ de décès à 5 ans pour les personnes avec un IMC stable à $35 \mathrm{~kg} / \mathrm{m}^{2}$ ) [24]. À noter que ces rapports de registre ne font pas la différence entre perte de poids désirée ou non. Il est donc tout à fait possible de penser que les personnes dont le pronostic est le plus altéré correspondent à celles qui présentent les comorbidités les plus importantes, comme les cancers par exemple, et donc à celles qui perdent du poids.

En post transplantation, les résultats sont aussi peu encourageants. Les registres américains de 2004-2014 montrent qu'il existe un lien entre une variation pondérale avant la transplantation, que ce soit une perte ou un gain de poids, une augmentation des pertes de greffon et une hausse de la mortalité [25]. Nous sommes toujours sur une variation pondérale constatée sans notion de perte volontaire ou non. En France, une cohorte de Heng et al. confirmait l'augmentation de la mortalité (HR 4,24 ; IC 95 \%, 1,32-13,6 ; $p=0,01)$ sans effet statistique sur la perte du greffon (HR 1,14; IC $95 \% 0,29-4,54 p=0,86$ ) après une perte de poids des personnes en situation d'obésité supérieure à $10 \%$. Ceci sans effet sur les complications chirurgicales [26].

\section{Chirurgie bariatrique avant transplantation rénale}

Malgré les chiffres précédents, plusieurs équipes se sont posées la question d'une chirurgie bariatrique avant la transplantation. Les cohortes qui en découlent nous placent dans le cadre d'une perte de poids volontaire et déterminée dans le temps. Yemini et al. montrent sur 24 chirurgies bariatriques pré-transplantation (IMC avant chirurgie de $41 \mathrm{~kg} / \mathrm{m}^{2}$ ) un taux de transplantation à 4 ans de 16/24 (66,6 \%) avec seulement deux décès sur liste. L'IMC au moment de la transplantation était de $28 \mathrm{~kg} / \mathrm{m}^{2}$. Les données sur les complications métaboliques sont aussi intéressantes avec des baisses importantes du nombre de patients ayant un diabète de type 2, une dyslipidémie ou une hypertension artérielle [27]. Bouchard et al. [28] ont publié une cohorte qui incluait des patients avec un IMC bien plus sévère à $51 \mathrm{~kg} / \mathrm{m}^{2}$ et donc présentant une contreindication pour la transplantation selon les recommandations de la HAS [9]. Pour ces patients normalement récusés, la perte en terme d'IMC après sleeve-gastrectomie a été de $9,8 \mathrm{~kg} / \mathrm{m}^{2} .20$ patients sur 32 ont été inscrits sur liste (63\%) et 14 (44\%) greffés à 1 an. Il y avait un décès sur liste. Si, comme pour l'autre étude, cette cohorte 
ne présente pas de bras contrôle, elle démontre la faisabilité de la transplantation rénale chez ces personnes avec un IMC supérieur à $50 \mathrm{Kg} / \mathrm{m}^{2}$ et sa relative sécurité. Néanmoins, les effectifs restent faibles et il serait intéressant d'aller plus loin sur l'étude de l'impact de la chirurgie bariatrique sur le temps d'attente sur liste.

La chirurgie bariatrique peut aussi représenter une sorte de contrat moral entre le patient et l'équipe soignante afin de limiter les risques. La chirurgie conditionne l'inscription sur liste de transplantation mais, une fois le geste réalisé, cette inscription se fait rapidement. Il serait, de même, possible de penser le couplage d'une chirurgie bariatrique avec un projet de donneur vivant. A ceci près que la chirurgie sur greffon issue de donneur vivant présente l'inconvénient d'un uretère plus court et donc d'une chirurgie techniquement plus compliquée. Les bénéfices attendus de la chirurgie bariatrique risquent donc d'être perdus par la complexification du geste opératoire.

En ce qui concerne la chirurgie par bypass, elle présente un risque de néphropathie à l'oxalate secondaire à l'hyperoxalurie, elle-même induite par ce type de chirurgie [29]. La technique par malabsorption est aussi une question majeure pour l'équilibre des immunosuppresseurs. Ces notions s'ajoutent aux complications déjà connues de ce type de chirurgie et n'en font pas la technique de premier choix des équipes de transplantation rénale.

La question des complications chirurgicales sur la transplantation rénale se pose aussi du côté des donneurs vivants. Plusieurs protocoles ont envisagé des programmes de perte de poids sur les donneurs de rein [30]. La question est bien entendu, ici, celle du rallongement, pour le receveur de la durée d'attente de la transplantation versus la diminution des complications chirurgicales chez le donneur. Ceci est un débat éthique propre à la transplantation de donneur vivant et nécessite plus de preuves.

\section{Programmes de réentraînement et prise en charge diététique}

Un certain nombre d'études se sont posées la question de l'efficacité des programmes de réentraînement physique chez les personnes en attente de transplantation. Les données sur les caractéristiques anthropométriques sont encourageantes. Si aucun lien direct n'est mis en évidence avec la survie, le lien avec l'augmentation de la masse musculaire est, lui, bien acquis. Cette augmentation de la masse musculaire est aussi liée, dans d'autres travaux, à une amélioration des conditions de vie et de survie en dialyse [31,32]. Quelques études ont également été menées sur des programmes de modification des habitudes de vie avec des effets intéressants sur les caractéristiques métaboliques posttransplantation, notamment en termes d'augmentation de la masse musculaire [33]. Ces travaux ne lèvent pas pour autant la question du risque pour les personnes sur liste d'attente de protocoles nutritionnels (de régimes ? de régimes restrictifs ?) avec risque de dénutrition, de sarcopénie et donc d'effet délétère au long cours.

La sarcopénie, même ajustée sur l'IMC, semble liée à une augmentation de la mortalité après la transplantation [34]. Les programmes de renforcement musculaire ont donc toute leur place sur l'ensemble du parcours des personnes en IRCT ayant un projet de transplantation puis en suivi post transplantation.

\section{L'IMC est-il le bon marqueur ? Quid du tour de taille ?}

Plusieurs équipes ont tenté de s'extraire de la notion d'IMC dans le pronostic des transplantations rénales. D'un point de vue chirurgical, c'est en effet le tablier abdominal et donc le tour de taille qui représente finalement l'obstacle anatomique au geste. Ainsi, une étude de Taha et al. qui ajuste le tour de taille sur l'IMC et qui montre que c'est là l'élément le plus en lien avec les complications chirurgi- cales de la transplantation rénale [35]. Dans une cohorte hongroise 1996 à 2012 de 993 personnes avec un suivi moyen post transplantation de 3,2 ans, Kovedsy et al. rapportent que l'IMC n'est pas un facteur de risque de mortalité sauf si on l'ajuste sur le tour de taille et il existe, alors, une corrélation inverse entre IMC et mortalité. Ainsi, l'IMC au-dessus de $30 \mathrm{~kg} / \mathrm{m}^{2}$ devient protecteur au contraire des IMC en dessous de $25 \mathrm{~kg} / \mathrm{m}^{2}$. Dans le même sens, le tour de taille n'était pas corrélé à la survie, sauf ajusté sur l'IMC où le groupe avec le tour de taille le plus important présentait la mortalité la plus importante.

En résumé, une personne avec un IMC important mais avec un faible tour de taille semble plutôt protégée [36]. Cela est corroboré par les résultats de l'équipe du CHU de Montpellier qui montrait dans un travail académique que le quartile des personnes avec le tour de taille le plus important présentait le taux de perte de greffon le plus important [37]. La description de cette cohorte permet de comprendre les limites des analyses des registres actuellement disponibles. Malgré un recrutement globalement exhaustif, ils n'avaient que $5,6 \%$ de personnes diabétiques et $0,8 \%$ de personnes avec un IMC au-dessus de $35 \mathrm{~kg} / \mathrm{m}^{2}$. Le quartile de personnes avec le tour de taille le plus important présentait un tour de taille supérieur à $98 \mathrm{~cm}$ chez les hommes et $90 \mathrm{~cm}$ chez les femmes. De fait les cohortes, surtout avant le tournant des années 2000, comportent peu de personnes avec un IMC supérieur à $30 \mathrm{~kg} / \mathrm{m}^{2}$, idem pour le tour de taille. Les patients analysés sont donc sélectionnés et peuvent induire des biais lorsque le clinicien se projette face à l'épidémiologie actuelle des personnes arrivant au stade de la suppléance rénale et donc de la question de l'inscription sur liste.

\section{Conclusion}

Si l'obésité présente des complications rénales propres, un IMC important semble protecteur en dialyse bien que les taux de mortalité restent très importants chez tous les patients suivant un traitement de suppléance artificielle. La transplantation rénale améliore cependant le pronostic des personnes quel que soit leur IMC. Un IMC élevé semble, cependant, être un frein majeur à l'accès à la transplantation alors que la proportion de personnes en situation d'obésité candidates à une inscription sur liste de transplantation rénale ne cesse d'augmenter dans les pays occidentaux. Par ailleurs, le lien entre IMC, mortalité post-transplantation et perte du greffon est loin d'être consensuel. Il existe une série de barrières, notamment sur les immunosuppresseurs que le développement de cohortes spécifiques devrait pouvoir en partie lever avec le temps. Cependant, c'est bien la problématique chirurgicale qui représente le couperet de la prise en charge. Le problème de l'abord chirurgical et notamment du tablier abdominal est aujourd'hui encore un frein. De fait, plus que l'IMC, outil de suivi controversé, les études devraient tendre vers une analyse du tour de taille comme facteur barrière pour la chirurgie, cela, au-delà de son implication connue dans l'inflammation chronique et le développement des maladies métaboliques. L'amaigrissement en dialyse, s'il est proposé aux patients, est particulièrement lié à une augmentation de la mortalité et doit réellement être mis dans la balance bénéficerisque. Dans ce contexte, la place de la chirurgie bariatrique peut être discutée au cas par cas. Le risque de la perte de poids est finalement à mettre en balance avec le projet d'inscription sur liste.

Néanmoins, le développement de nouvelles techniques et des publications récentes sur l'utilisation du robot pour cette chirurgie est peut-être l'espoir qu'attendent les patients en attente d'inscription sur liste. Quoi qu'il en soit, il existe suffisamment de questions métaboliques pour que les équipes de nutrition, et de diététique de manière générale, soient impliquées dans le suivi des personnes vivant en situation d'obésité et en attente de transplantation rénale. 


\section{Protection des droits des sujets humains et animaux}

Les auteurs déclarent que les travaux décrits n'ont pas impliqué d'expérimentations sur les patients, sujets ou animaux.

\section{Consentement éclairé et confidentialité des données}

Les auteurs déclarent que les travaux décrits n'impliquent aucun patient ou sujet.

\section{Déclaration de liens d'intêrêts}

Les auteurs déclarent ne pas avoir de liens d'intérêts.

\section{Financement}

Cette étude n'a reçu aucun financement spécifique d'une agence publique, commerciale ou à but non lucratif.

\section{Contribution et responsabilité des auteurs}

L'ensemble des auteurs attestent du respect des critères de l'International Committee of Medical Journal Editors (ICMJE) en ce qui concerne leur contribution à l'article. Les contributions des auteurs se sont réparties comme suit : Rédacteur principal : Jeanbaptiste Bonnet. L'ensemble des autres auteurs ont relus complété et validé le contenu de l'article.

\section{Références}

[1] Prélèvement sur donneur décédé en état de mort encéphalique [Internet] Rapport 2017; 2018. Available from: https://www.agence-biomedecine.fr/ annexes/bilan2017/donnees/organes/01-prelevement/pdf/prelevement.pdf [cited 2020 Aug 18].

[2] Synthese_irct_vf.pdf [Internet]; 2014. Available from: https://www.hassante.fr/upload/docs/application/pdf/2014-11/synthese_irct_vf.pdf [cited 2020 Jun 20].

[3] Krishnan N, Higgins R, Short A, Zehnder D, Pitcher D, Hudson A, et al. Kidney transplantation significantly improves patient and graft survival irrespective of BMI: a cohort study: high bmi and kidney transplantation. Am J Transplant 2015;15(9):2378-86.

[4] Gill JS, Lan J, Dong J, Rose C, Hendren E, Johnston O, et al. The survival benefit of kidney transplantation in obese patients. Am J Transplant 2013;13 (8):2083-90.

[5] Segev DL, Simpkins CE, Thompson RE, Locke JE, Warren DS, Montgomery RA. Obesity Impacts Access to Kidney Transplantation. J Am Soc Nephrol 2008:19(2):349-55.

[6] Friedman AN, Miskulin DC, Rosenberg IH, Levey AS. Demographics and trends in overweight and obesity in patients at time of kidney transplantation. Am J Kidney Dis 2003:41(2):480-7.

[7] Rapport_rein2013.pdf [Internet]; 2015. Available from: https://www.agencebiomedecine.fr/IMG/pdf/rapport_rein2013.pdf [cited 2020 Jun 18].

[8] Toapanta-Gaibor NG, Suñer-Poblet M, Cintra-Cabrera M, Pérez-Valdivia MÁ, Suárez-Benjumea A, Gonzalez-Roncero FM, et al. Reasons for noninclusion on the kidney transplant waiting list: analysis in a set of hemodialysis centers. Transplant Proc 2018:50(2):553-4.

[9] Rbp_argumentaire_greffe_renale_vd_mel.pdf [Internet]; 2015. Available from: https://www.has-sante.fr/upload/docs/application/pdf/2015-12/rbp_ argumentaire_greffe_renale_vd_mel.pdf [cited 2020 Jun 20].

[10] Suresh A, Robinson L, Milliron BJ, Leonbeerg K, McAdams-DeMarco M, Earthman C, Klassen A, Harhay N. Approaches to obesity management in dialysis settings: renal dietitian perspectives. J Renal Nutr 2020.

[11] Leavey SF, McCullough K, Hecking E, Goodkin D, Port FK, Young EW. Body mass index and mortality in "healthier" as compared with "sicker" haemodialysis patients: results from the Dialysis Outcomes and Practice Patterns Study (DOPPS). Nephrol Dial Transplant 2001;16(12):2386-94.

[12] Aalten J, Christiaans MH, de Fijter H, Hené R, Homan van der Heijde J, Roodnat J, et al. The influence of obesity on short- and long-term graft and patient survival after renal transplantation. Transpl Int 2006;19(11):901-7.
[13] Chang SH, Coates PTH, McDonald SP. Effects of body mass index at transplant on outcomes of kidney transplantation. Transplantation 2007:84(8):981-7.

[14] Singh D, Lawen J, Alkhudair W. Does pretransplant obesity affect the outcome in kidney transplant recipients? Transplant Proc 2005;37(2):717-20.

[15] Humar A, Ramcharan T, Denny R, Gillingham KJ, Payne WD, Matas AJ. Are wound complications after a kidney transplant more common with modern immunosuppression? Transplantation 2001;72(12):1920-3.

[16] Spaggiari M, Lendacki FR, Di Bella C, Giulianotti PC, Benedetti E, Oberholze J, et al. Minimally invasive, robot-assisted procedure for kidney transplantation among morbidly obese: positive outcomes at 5 years post-transplant. Clin Transplant 2018:32(11):e13404

[17] Hameed AM, Yao J, Allen RD, Hawthorne WJ, Pleass HC, Lau H. The evolution of kidney transplantation surgery into the robotic era and its prospects for obese recipients. Transplantation 2018:102(10):1650-65.

[18] D’Agati VD, Chagnac A, de Vries APJ, Levi M, Porrini E, Herman-Edelstein M, et al. Obesity-related glomerulopathy: clinical and pathologic characteristics and pathogenesis. Nat Rev Nephrol 2016;12(8):453-71.

[19] Wang Y, Chen X, Song Y, Caballero B, Cheskin LJ. Association between obe sity and kidney disease: a systematic review and meta-analysis. Kidney Int 2008;73(1):19-33.

[20] El-Agroudy AE, Wafa EW, Gheith OE, Shehab El-Dein AB, Ghoneim MA. Weight gain after renal transplantation is a risk factor for patient and graft outcome. Transplantation 2004;77(9):1381-5.

[21] Sood A, Hakim DN, Hakim NS. Consequences of recipient obesity on postoperative outcomes in a renal transplant: a systematic review and meta-analysis. Exp Clin Transpl 2016;14(2):121-8

[22] Bayés B, Granada ML, Pastor MC, Lauzurica R, Salinas I, Sanmartí A, et al. Obesity, adiponectin and inflammation as predictors of new-onset diabetes mellitus after kidney transplantation. Am J Transplant 2007;7(2):416-22.

[23] Flabouris K, Chadban S, Ladhani M, Cervelli M, Clayton P. Body mass index, weight-adjusted immunosuppression and the risk of acute rejection and infection after kidney transplantation: a cohort study. Nephrol Dial Transplant $2019 ; 34(12): 2132-43$

[24] Brilleman SL, Moreno-Betancur M, Polkinghorne KR, McDonald SP, Crowther MJ, Thomson J, et al. Changes in body mass index and rates of death and transplant in hemodialysis patients: a latent class joint modeling approach. Epidemiology 2019;30(1):38-47.

[25] Harhay MN, Ranganna K, Boyle SM, Brown AM, Bajakian T, Mizrahi LBL, et al. Association between weight loss before deceased donor kidney transplantation and post-transplantation outcomes. Am J Kidney Dis 2019;74(3):361-72.

[26] Heng A-E, Pereira B, Fervenza F, Boirie Y, Prieto M. Renal transplant in obese patients and impact of weight loss before surgery on surgical and medical outcomes: a single-center cohort study. Exp Clin Transplant 2018.

[27] Yemini R, Nesher E, Carmeli I, Winkler J, Rahamimov R, Mor E, et al. Bariatric surgery is efficacious and improves access to transplantation for morbidly obese renal transplant candidates. Obes Surg 2019;29(8):2373-80.

[28] Bouchard P. Tchervenkov J, Demyttenaere S, Court O, Andalib A. Safety and efficacy of the sleeve gastrectomy as a strategy towards kidney transplantation. Surg Endosc 2020;34(6):2657-64.

[29] Troxell ML, Houghton DC, Hawkey M, Batiuk TD, Bennett WM. Enteric oxalate nephropathy in the renal allograft: an underrecognized complication of bariatric surgery. Am J Transplant 2013;13(2):501-9.

[30] Montgomery JR, Telem DA, Waits SA. Bariatric surgery for prospective living kidney donors with obesity? Am J Transplant 2019;19(9):2415-20

[31] Masajtis-Zagajewska A, Nowicki M. Effects of a structured physical activity program on habitual physical activity and body composition in patients with chronic kidney disease and in kidney transplant recipients. Exp Clin Transplan 2019;17(2):155-64.

[32] Calella P, Hernández-Sánchez S, Garofalo C, Ruiz JR, Carrero JJ, Bellizzi V. Exercise training in kidney transplant recipients: a systematic review. J Nephrol 2019;32(4):567-79.

[33] Sharif A, Moore R, Baboolal K. Influence of lifestyle modification in renal transplant recipients with postprandial hyperglycemia. Transplantation 2008;85(3):353-8

[34] Jean sébastien S. Impact de l'atrophie musculaire et de l'obésité sur la survie en transplantation rénale [MD Thesis]. Université Montpellier; 2008. p. 1.

[35] Taha M, Davis NF, Power R, Mohan P, Forde J, Smyth G, et al. Increased midabdominal circumference is a predictor for surgical wound complications in kidney transplant recipients: a prospective cohort study. Clin Transplant 2017;31(5):e12960

[36] Kovesdy CP, Czira ME, Rudas A, Ujszaszi A, Rosivall L, Novak M, et al. Body mass index, waist circumference and mortality in kidney transplant recipients: obesity and mortality in kidney transplant. Am J Transplant 2010;10(12) 2644-51.

[37] Mathilde H. Impact de l'obésité sur la survie après transplantation rénale : résultats d'une cohorte montpelliéraine [MD Thesis]. Université de Montpellier; 2017. 